\title{
THE ZEROS OF A CERTAIN FAMILY OF TRINOMIALS
}

\author{
by KARL DILCHER, JAMES D. NULTON and KENNETH B. STOLARSKY
}

(Received 2 August, 1990)

1. Introduction. Many of the classical inequalities of analysis can be written in the form $P(x) \geq 0$ for $x \in I$ or $P(x)>0$ for $x \in I^{\prime}$, where $P(x)$ is a polynomial and $I^{\prime} \subset I$ are certain intervals on the real line. This gives rise to the question of where the zeros of $P(x)$ are located. For example, if $f$ is a polynomial with real zeros, then an inequality of Laguerre [8, p.171 f.] asserts that

$$
P(x)=\left(f^{\prime}(x)\right)^{2}-f(x) f^{\prime \prime}(x) \geq 0
$$

for all $x$. A detailed study of the zeros of this particular $P(x)$ has been made [5].

A more fundamental role in the study of inequalities is played by the trinomial

$$
T(x):=m x^{n}-n x^{m}+n-m, \quad n>m>0,
$$

and the corresponding inequality $T(x)>0$ for $0<x<1$ (see, e.g., [1, pp. 12-15] or [7, pp. 39-42]). For example, upon replacing $x$ by $(x / y)^{1 / n}$ (with, say, $y>x$ ) and $m / n$ by $\lambda$, we obtain the weighted arithmetic-geometric mean inequality

$$
\lambda x+(1-\lambda) y \geq x^{\lambda} y^{1-\lambda} .
$$

The zero distribution of $T(x)$ in (1.1) does not seem to have been studied previously; this will be done in Section 2 .

If in (1.2) $y$ is replaced by 1 , we obtain the inequality

$$
1-\lambda+\lambda x-x^{\lambda} \geq 0, \quad 0<x<1, \quad 0<\lambda<1 .
$$

Next we replace $x$ by $e^{u}$, to obtain the exponential polynomial $P(u):=1-\lambda+\lambda e^{u}-e^{\lambda u}$. We replace now $e^{a u}$ by $(1+a u / n)^{n}$; this is in accordance with the program of "reductionism", as outlined in [15] (see also [16] and [17]). With the new variable $z=1+u / n$ we finally obtain the polynomial

$$
p_{n}(z):=\lambda z^{n}+1-\lambda-(\lambda z+1-\lambda)^{n} .
$$

Note that $p_{n}(z)$ tends to the left side of (1.3) as $n \rightarrow \infty$.

The main purpose of this paper is to show that the zeros of $p_{n}(z)$ depend on $\lambda$ in an unexpected way:

THEOREM 1.1. If $\lambda$ is fixed and $n$ is sufficiently large, then $p_{n}(z)$ has a zero inside the unit circle if and only if $\lambda$ is not the reciprocal of a positive integer.

This result was announced in [11]; here we shall provide a proof and show that one direction of Theorem 1.1 is true for all $n$. In particular, we shall show in Section 3 that $p_{n}(z)$ has no zero inside the unit circle for any $\lambda$, provided that $n \leq \frac{5}{51 \lambda}+1$. In Section 4 it will be shown that $p_{n}(z)$ does have zeros inside the unit circle if $\lambda$ is not the reciprocal of a positive integer and $n$ is sufficiently large. The most difficult part is to show that $p_{n}(z)$ has no zeros inside the unit circle if $\lambda$ is the reciprocal of a positive integer. This case will be dealt with in Sections 5-8. 
2. Zeros of the trinomial $T(x)$. In this section we study the distribution of zeros of the trinomial $T(x)$ in (1.1). We begin with a result on a more general class of trinomials. In what follows, let $U$ denote the unit circle.

Theorem 2.1. Let $a>b>0$ be real numbers and $n>m>0$ be integers. Then the number of zeros of

$$
P(z):=b z^{n}-a z^{m}+a-b
$$

strictly inside $U$ is $m-\operatorname{gcd}(m, n)$ if $a / b \geq n / m$, and $m$ if $a / b<n / m$.

We prove this result later in this section. Let us remark that other results on similar trinomials can be found, e.g., in [3], [13], or [14].

COROLlaRY 2.2. If $n>m>0$ are two relatively prime integers, then the trinomial $T(x)$ in (1.1) has $m-1$ zeros strictly inside $U, n-m-1$ zeros strictly outside $U$, and a double zero at $z=1$.

Proof. The first statement is immediate from Theorem 2.1, and the second statement is obtained from the first one by considering the inverse polynomial

$$
z^{n} T(1 / z)=(n-m) z^{n}-n z^{n-m}+n-(n-m) .
$$

The third statement becomes clear by considering the first and second derivatives of $T(z)$.

We note that the trinomials $T(z)$ were recently considered in [10], for the special case $m=1$. Very exact bounds on the moduli and arguments of the zeros were given in this case. Here we shall prove a weaker result for all $m, 1 \leq m \leq n-1$. We also note that applications to the theory of signal processing are indicated in [10], for $m=1$.

Proposition 2.3. For $n \geq 3$ the zeros of $(z-1)^{-2} T(z)$ lie in the following annuli:

$$
1+(n-2)^{-1} \leqslant|z| \leq[2(n-1)]^{1 /(n-1)} \text { for } m=1 ;
$$

(b) $\max \left\{(2 m)^{-1 / m}, 1-\frac{1}{n-m} \sqrt{2 n / m}\right\} \leq|z|$

$$
\leq \min \left\{[2(n-m)]^{1 /(n-m)}, 1+\frac{1}{m} \sqrt{2 n /(n-m)}\right\} \text { for } 2 \leq m \leq n-2 ;
$$

(c)

$$
[2(n-1)]^{-1 /(n-1)} \leq|z| \leq 1-(n-1)^{-1} \text { for } m=n-1 .
$$

Proof. (a) is quoted in [10]. (c) follows from (a) by considering the inverse polynomial (2.2). To prove (b), we apply two different methods for finding zeros of polynomials to $T(z)$.

By Cauchy's theorem [9, Theorem 27.1], the maximum modulus of the zeros of $T(z)$ is at most equal to the positive zero of

$$
f(x):=m x^{n}-n x^{m}-(n-m) .
$$

Since $f(x)$ is increasing for $x \geq 1$ and $f(1)=2 m-2 n<0$, it suffices to find an $x_{0}>1$ such that $f\left(x_{0}\right)>0$. Let us consider

$$
x_{0}:=[2(n-m)]^{1 /(n-m)} \text {. }
$$


Then

$$
\begin{aligned}
f\left(x_{0}\right) & =x_{0}^{m}\left(m x_{0}^{n-m}-n\right)-n+m=x_{0}^{m}(2 m(n-m)-n)-n+m \\
& >(2 m(n-m)-n)-n+m=2(m-1) n-2 m^{2}+m \\
& \geq 2(m-1)(m+1)-2 m^{2}+m \geq 0,
\end{aligned}
$$

which proves half of the right-hand inequality in (b).

For the second half, we use Rouché's theorem [9, p.2]. Let $z=(1+\delta) e^{i t}$ with $\delta>0$ and $t \in \mathbb{R}$. Then since

$$
\left|z^{n}-1\right| \geq(1+\delta)^{n}-1,\left|z^{m}-1\right| \leq(1+\delta)^{m}+1,
$$

we have

$$
\left|m\left(z^{n}-1\right)\right|>\left|n\left(z^{m}-1\right)\right|
$$

when

$$
m(1+\delta)^{n}-m>n(1+\delta)^{m}+n
$$

or

$$
(1+\delta)^{m}\left\{m(1+\delta)^{n-m}-n\right\}>n+m .
$$

Using the estimate $(1+\delta)^{k}>1+k \delta$ for $\delta>0$ and $k \geq 2$, we find that this last inequality holds when $(1+m \delta)\{m \delta-1\} \geq(n+m) /(n-m)$, or equivalently

$$
\delta \geq \delta_{0}:=(\sqrt{2 n /(n-m)}) / m .
$$

Hence by Rouche's theorem with (2.3), $T(z)$ has the same number of zeros inside the circle $|z|=1+\delta_{0}$ as $m\left(z^{n}-1\right)$, namely all $n$ zeros. This proves the right inequality of (b). We get the left inequality now by considering the inverse polynomial (2.2) and applying the right inequality; here we use the fact that $\left(1+\delta_{0}\right)^{-1} \geq 1-\delta_{0}$. The proof is now complete.

The next proposition states that the zeros of $T(z)$ are quite evenly distributed inside the (generally narrow) annuli of Proposition 2.3.

Proposition 2.4. The $m-1$ "inner zeros" of $T(z)$ lie in the neighbourhood of the $m$-th roots of unity different from 1 , and the $n-m-1$ "outer zeros" lie in the neighbourhood of the $(n-m)$ th roots of unity different from 1 . More explicitly: let $m$ and $\varepsilon>0$ be fixed. Then there is an $N$ such that the inner zeros lie within distance $\varepsilon$ of the $m$-th roots of unity for all $n>N$. If $n-m$ and $\varepsilon>0$ are fixed, then there is an $M$ such that the outer zeros lie within $\varepsilon$ of the $(n-m)$ th roots of unity for all $n>M$.

Proof. For $\delta>0$, consider

$$
T_{\delta}(z):=m z^{n}-n z^{m}+n(1-\delta)-m .
$$

If $\delta>0$ is sufficiently small, then the $m-1$ inner zeros of $T(z)$ will still correspond to inner zeros of $T_{\delta}(z)$. In addition, the double zero at $z=1$ of $T(z)$ will split into an inner and an outer real zero of $T_{\delta}(z)$. Now we observe that

$$
T_{\delta}(z) / n \rightarrow-z^{m}+1-\delta \text { for }|z| \leq 1 \text {, as } n \rightarrow \infty \text {; }
$$


hence by the theorem of Hurwitz (see, e.g., [9, p. 4]) the $m$ inner zeros tend to $(1-\delta)^{1 / m} \varepsilon_{j}$, where the $\varepsilon_{j}, j=1, \ldots, m$, are the $m$-th roots of unity. Since $\delta$ can be chosen arbitrarily small, this proves the first part of the proposition. For the second part, we repeat this argument for the inverse polynomial $z^{n} T(1 / z)$ in (2.2).

Now we proceed to the proof of Theorem 2.1. We need the following two lemmas.

LEMMA 2.5. If $b>a>0$ and $p, q$ are all real, then

$$
b z^{q}-a z^{p}=b-a, \quad z=e^{i \theta}
$$

implies $p \theta \equiv q \theta \equiv 0(\bmod 2 \pi)$.

Proof. The length of the line segment joining a point on $|z|=a$ to a point on $|z|=b$ can equal $b-a$ only if the two points have the same argument.

LEMMA 2.6. Let $n>m>0$ be integers. If

$$
A z^{n}+B z^{m}+C=0, \text { with }|B|>|A|+|C|,
$$

then there are exactly $m$ zeros of (2.4) strictly inside $U$.

Proof. This is an immediate consequence of Rouché's theorem.

Proof of Theorem 2.1. Let $\varepsilon>0$ and consider

$$
P_{\varepsilon}(z)=b z^{n}-a z^{m}+a-b-\varepsilon .
$$

By Lemma 2.6, for small positive $\varepsilon$ this has exactly $m$ zeros strictly inside $U$, say $\alpha_{1}, \ldots, \alpha_{m}$. As $\varepsilon \rightarrow 0$, some of these may tend to $U$. If

$$
\alpha_{j} \rightarrow e^{i \theta_{i}}=e^{i \theta}, \quad \theta \in \mathbb{R},
$$

then by Lemma $2.5, n \theta \equiv m \theta(\bmod 2 \pi)$. Hence $e^{i \theta}$ is both an $m$-th and an $n$-th root of unity, and therefore a $d$-th root of unity, where $d=\operatorname{gcd}(m, n)$. Conversely, if $\omega^{d}=1$, then $P(\omega)=0$.

Now suppose that $\alpha_{j} \rightarrow \omega$, where $\omega^{d}=1$. Since

$$
P^{\prime}(z)=n b z^{n-1}-a m z^{m-1},
$$

the root $\omega$ has multiplicity one unless $m / n=b / a$, in which case it has multiplicity two. Now set

$$
\beta=r \omega, \quad 0<r<\infty,
$$

and

$$
h(r):=P_{\varepsilon}(\beta)=b r^{n}-a r^{m}+a-b-\varepsilon .
$$

It is easy to see that $h(r)$ has two positive zeros, while $h^{\prime}(r)$ has only one positive zero $r^{\prime}$; also,

$$
r^{\prime}>1 \text { if } a / b>n / m
$$

while

$$
r^{\prime}<1 \text { if } a / b<n / m \text {. }
$$


In both these cases $h(r)$ has a zero $r_{0}$ between 0 and 1 , and for $r>r_{0}$ the function $h(r)$ remains negative until $r>r_{0}$, where of course $r^{\prime}$ is independent of the value of $\varepsilon$. Thus in case (2.5) it is an inside zero that goes to $U$ as $\varepsilon \rightarrow 0$, while in (2.6) it is an outside zero. In the case of a double zero, it is clear that both an inside and an outside zero are coming together. The case $a / b=n / m$ is resolved by a continuity argument, and the result follows.

3. Zeros of $p_{n}(z)$ for small $n$. We now turn to the study of the sequence of polynomials

$$
p_{n}(z):=\lambda z^{n}+1-\lambda-(\lambda z+1-\lambda)^{n} .
$$

The following result shows that for small $n$ (the size depending on $\lambda$ ), the distribution of zeros of $p_{n}(z)$ does not depend on the arithmetic nature of $\lambda$.

THEOREM 3.1. If $0<\lambda<1$ is any fixed real number, then $p_{n}(z)$ has no zero inside $U$ for $n \leq \frac{5}{51 \lambda}+1$.

Proof. 1. Let $\lambda=1 / q$, where $q>1$ is (in this section only) an arbitrary real number. Then

$$
q^{n} p_{n}(z)=q^{n-1}\left(z^{n}+q-1\right)-(z+q-1)^{n} .
$$

Using first a binomial expansion for $(z+q-1)^{n}$ and then the identity

$$
z^{n}-1-n(z-1)=(z-1)^{2}\left[z^{n-2}+2 z^{n-3}+\ldots+(n-1)\right]
$$

we obtain

$$
q^{n} p_{n}(z)=\left[z^{n}-1-n(z-1)\right] q^{n-1}-\sum_{k=0}^{n-2}\left(\begin{array}{l}
n \\
k
\end{array}\right)(z-1)^{n-k} q^{k}
$$

and therefore

$$
\begin{aligned}
\frac{q^{n} p_{n}(z)}{(z-1)^{2}} & =\left[z^{n-2}+2 z^{n-3}+\ldots+(n-1)\right] q^{n-1}-\sum_{k=0}^{n-2}\left(\begin{array}{l}
n \\
k
\end{array}\right)(z-1)^{n-k-2} q^{k} \\
& =: P(z) q^{n-1}-Q(z) .
\end{aligned}
$$

If we can show that

$$
\left|P\left(e^{i \theta}\right)\right| q^{n-1}>\left|Q\left(e^{i \theta}\right)\right| \text { for } 0 \leq \theta \leq 2 \pi,
$$

then by Rouché's theorem, (3.2) and hence $p_{n}(z)$ has the same number of zeros inside the unit circle as does $P(z)$; but by the Kakeya-Eneström theorem [12, Problem III.22], this number is zero. This proves the theorem, provided that we can show (3.3) for $n \leq \frac{5}{51} q+1$.

2. We begin by estimating $\left|Q\left(e^{i \theta}\right)\right|$. Using

$$
\left(\begin{array}{l}
n \\
k
\end{array}\right)=\frac{n(n-1)}{(n-1-k)(n-k)}\left(\begin{array}{c}
n-2 \\
k
\end{array}\right) \leq \frac{n(n-1)}{2}\left(\begin{array}{c}
n-2 \\
k
\end{array}\right)
$$

for $k \leq n-2$, and

$$
\left|e^{i \theta}-1\right|=(2-2 \cos \theta)^{1 / 2}=2 \sin \frac{\theta}{2}
$$


we obtain

$$
\left|Q\left(e^{i \theta}\right)\right| \leq \sum_{k=0}^{n-2}\left(\begin{array}{l}
n \\
k
\end{array}\right)\left(2 \sin \frac{\theta}{2}\right)^{n-k-2} q^{k} \leq \frac{n(n-1)}{2} \sum_{k=0}^{n-2}\left(\begin{array}{c}
n-2 \\
k
\end{array}\right)\left(2 \sin \frac{\theta}{2}\right)^{n-2-k} q^{k},
$$

that is,

$$
\left|Q\left(e^{i \theta}\right)\right| \leq \frac{n(n-1)}{2}\left(q+2 \sin \frac{\theta}{2}\right)^{n-2} .
$$

3. To estimate $\left|P\left(e^{i \theta}\right)\right|$, we first consider $0 \leq \theta<\pi / n$. With (3.1) and (3.4) we get

$$
\begin{aligned}
\left|P\left(e^{i \theta}\right)\right| & =\left|\frac{e^{i n \theta}-1-n\left(e^{i \theta}-1\right)}{\left(e^{i \theta}-1\right)^{2}}\right| \\
& =\frac{1}{2 \sin ^{2}(\theta / 2)}\left|\left(\cos \frac{n-1}{2} \theta+i \sin \frac{n-1}{2} \theta\right) \sin \frac{n \theta}{2}-n \sin \frac{\theta}{2}\right| .
\end{aligned}
$$

Since $|z| \geq|\operatorname{Im} z|$ for all complex $z$, we have

$$
\left|P\left(e^{i \theta}\right)\right| \geq \frac{1}{2 \sin ^{2}(\theta / 2)}\left|\sin \frac{n-1}{2} \theta \sin \frac{n \theta}{2}\right| .
$$

We note that for $0 \leq n \theta / 2 \leq \pi / 2$ we have

$$
\left|\sin \frac{n \theta}{2}\right| \geq \frac{2}{\pi} \frac{n \theta}{2}=\frac{n \theta}{\pi},
$$

while $0 \leq \sin (\theta / 2) \leq \theta / 2$, so that with (3.6) we get

$$
\left|P\left(e^{i \theta}\right)\right| \geq \frac{2}{\pi^{2}} n(n-1) \text { for } 0 \leq \theta \leq \pi / n .
$$

4. In the case $\pi / n \leq \theta \leq \pi$ we rewrite

$$
\left|P\left(e^{i \theta}\right)\right|=\left|\frac{n-\left(1+e^{i \theta}+\ldots+e^{i(n-1) \theta}\right)}{e^{i \theta}-1}\right| .
$$

Let $n$ be fixed and $K$ be such that $2^{K} \leq n$ but $2^{K+1}>n$. Denote $M:=2^{K}$; then

$$
\frac{n}{2}<M \leq n \text {. }
$$

Now fix a $k, 1 \leq k \leq K$; we note that with $m:=2^{k}$ we have

$$
\begin{aligned}
\left|1+e^{i \theta}+\ldots+e^{i(M-1) \theta}\right| \leq & \left|1+e^{i \theta}+\ldots+e^{i(m-1) \theta}\right|+\left|e^{i m \theta}+\ldots+e^{i(2 m-1) \theta}\right|+\ldots \\
& +\left|e^{i(M-m) \theta}+\ldots+e^{i(M-1) \theta}\right| \\
= & 2^{K-k}\left|1+e^{i \theta}+\ldots+e^{i(m-1) \theta}\right| \\
= & \frac{M}{m}\left|\frac{e^{i m \theta}-1}{e^{i \theta}-1}\right| .
\end{aligned}
$$


Now

$$
\begin{aligned}
\mid n & -\left(1+e^{i \theta}+\ldots+e^{i(n-1) \theta}\right) \mid \\
& \geq n-\left|1+e^{i \theta}+\ldots+e^{i(M-1) \theta}\right|-\left|e^{i M \theta}\right|-\ldots-\left|e^{i(n-1) \theta}\right| \\
& \geq M-\frac{M}{m}\left|\frac{e^{i m \theta}-1}{e^{i \theta}-1}\right|,
\end{aligned}
$$

so that

$$
\left|P\left(e^{i \theta}\right)\right| \geq \frac{M}{\left|e^{i \theta}-1\right|^{2}}\left\{\left|e^{i \theta}-1\right|-\frac{1}{m}\left|e^{i m \theta}-1\right|\right\}
$$

and, with (3.4),

$$
\left|P\left(e^{i \theta}\right)\right| \geq \frac{2 M}{4 \sin ^{2} \frac{\theta}{2}}\left\{\sin \frac{\theta}{2}-\frac{1}{m} \sin \frac{m \theta}{2}\right\}
$$

Using Taylor expansions, we find

$$
\sin \frac{\theta}{2}-\frac{1}{m} \sin \frac{m \theta}{2} \geq \frac{\theta}{2}-\frac{1}{6}\left(\frac{\theta}{2}\right)^{3}-\frac{1}{m}\left[\frac{m \theta}{2}-\frac{1}{6}\left(\frac{m \theta}{2}\right)^{3}+\frac{1}{120}\left(\frac{m \theta}{2}\right)^{5}\right]
$$

so that with $\sin (\theta / 2) \leq \theta / 2$ we get

$$
\left|P\left(e^{i \theta}\right)\right| \geq \frac{M m^{2}}{24} \theta\left[1-\frac{1}{m^{2}}-\frac{(m \theta)^{2}}{80}\right] .
$$

First, let $m \geq 4$ and

$$
\frac{\pi}{2 m} \leq \theta \leq \frac{\pi}{m}
$$

then

$$
1-\frac{1}{m^{2}}-\frac{(m \theta)^{2}}{80} \geq 1-\frac{1}{16}-\frac{\pi^{2}}{80}>\frac{48}{59}
$$

Furthermore, with (3.11) we get $m \theta \geq \pi / 2$, and with (3.8) and $m \geq 4$ we have $M m \geq 4 M>2 n$; so (3.10) implies

$$
\left|P\left(e^{i \theta}\right)\right| \geq \frac{2 \pi}{59} n
$$

This holds for each interval (3.11) with $4 \leq m \leq M$, and thus for the interval $\pi / 2 M \leq \theta \leq \pi / 4$. Since $2 M>n$ and $2 \pi / 59 \leq 2(n-1) / \pi^{2}$ for $n \geq 2,(3.7)$ shows that (3.12) holds for all $0 \leq \theta \leq \pi / 4$. 
5. For $\pi / 4 \leq \theta \leq \pi$, we use (3.9) with $m=2$ to get

$$
\begin{aligned}
\left|P\left(e^{i \theta}\right)\right| & \geq \frac{M(1-\cos (\theta / 2))}{2 \sin (\theta / 2)}=M \frac{1-\left(1-\sin ^{2}(\theta / 2)\right)^{1 / 2}}{2 \sin (\theta / 2)} \\
& \geq M \frac{1-\left(1-\frac{1}{2} \sin ^{2} \frac{\theta}{2}\right)}{2 \sin (\theta / 2)}
\end{aligned}
$$

and therefore, with (3.8),

$$
\left|P\left(e^{i \theta}\right)\right| \geq \frac{n}{8} \sin \frac{\theta}{2} \text { for } \pi / 4 \leq \theta \leq \pi .
$$

6. Consider now $\pi / 4 \leq \theta \leq \pi$. We see from (3.5) and (3.13) that (3.3) holds when

$$
q \frac{\sin (\theta / 2)}{n-1}>4\left(1+\frac{2 \sin (\theta / 2)}{q}\right)^{n-2} \text {. }
$$

It is easy to verify that the left-hand side of (3.14) grows faster in $\theta$ than the right-hand side for $0 \leq \theta \leq \pi$, when $q \geq 4 n$. Hence, if we set $a:=\sin (\pi / 8)$, then (3.14) holds for $\pi / 4 \leq \theta \leq \pi$ when

$$
\frac{q}{n-1}>\frac{4}{a}\left(1+\frac{2 a}{q}\right)^{n-1} .
$$

If we denote $d:=q /(n-1) a$, then the right-hand side of $(3.15)$ is less than $(4 / a) \exp (2 / d)$, and (3.15) holds when $d>\left(4 / a^{2}\right) \exp (2 / d)$. This is the case for $d \geq 26.58$, which in turn holds when $q \geq 51(n-1) / 5$. This verifies (3.3) for $\pi / 4 \leq \theta \leq \pi$.

For $0 \leq \theta \leq \pi / 4$, we note that by (3.12) and (3.5), (3.3) holds when

$$
\frac{q}{n-1} \geq \frac{59}{4 \pi}\left(1+\frac{2 a}{q}\right)^{n-1} \text {. }
$$

But this follows from (3.15) since $59 / 4 \pi<4 / a$. The proof is now complete since by symmetry it suffices to consider $0 \leq \theta \leq \pi$.

Remark. From (3.2) we can easily obtain a weaker version of Theorem 3.1: for fixed $n, p_{n}(z)$ has no zero inside $U$ for all $\lambda$ sufficiently small.

Indeed, it is obvious from (3.2) that

$$
q p_{n}(z)(z-1)^{-2} \rightarrow z^{n-2}+2 z^{n-3}+\ldots+(n-1) \text { as } q \rightarrow \infty .
$$

But the polynomial to the right has all zeros in the annulus $1+1 /(n-2) \leq|z| \leq 2$, by the Kakeya-Eneström theorem [12, Problem III.23]. Hence by Hurwitz's theorem, for any $r$ with $1 \leq r<1+1 /(n-2)$ the zeros of $p_{n}(z)$ will lie outside a circle of radius $r$ if $q$ is sufficiently large. See also the remark at the end of Section 5.

4. $\lambda$ not an integer reciprocal. In this section we establish the sufficiency part of Theorem 1.1.

THeOrem 4.1. If $0<\lambda<1$ is given and $1 / \lambda$ is not an integer, then $p_{n}(z)$ has a zero inside the unit circle if $n$ is sufficiently large. 
The proof rests on a lemma which follows from a well-known result on exponential polynomials.

Lemma 4.2. If $\alpha>1$ and $\alpha$ is not an integer, then

$$
h(s):=e^{\alpha s}-\alpha e^{s}+\alpha-1
$$

has infinitely many zeros with negative real part.

Proof. First assume $\alpha=n / m$, where $n>m>0$ are two relatively prime integers. By Theorem 2.1 the trinomial $P(z)$ of (2.1) has $m-1$ zeros strictly inside $U$ (note that $m>1$ since $\alpha$ is not an integer); let $x_{0}=r e^{i \theta}$ be one of these. Then for any integer $k$,

$$
\exp \{\alpha(m \log r-\operatorname{im} \theta+2 \pi \operatorname{im} k)\}-\alpha \exp (m \log r+\operatorname{im} \theta+2 \pi \operatorname{im} k)+\alpha-1=0 ;
$$

this proves the lemma for rational $\alpha$.

Now assume that $\alpha$ is irrational. Let

$$
2 \alpha=\left[a_{0}, a_{1}, a_{2}, \ldots\right]
$$

be the continued fraction expansion of $2 \alpha$, and write

$$
\left[a_{0}, a_{1}, \ldots, a_{m}\right]=P_{m} / Q_{m}, \quad \operatorname{gcd}\left(P_{m}, Q_{m}\right)=1 .
$$

From the elementary properties of continued fractions it follows that at least one of $P_{m}, P_{m+1}$ is odd for $m \geq 0$. Thus for infinitely many positive integers $Q$ there is an odd $P$ such that

$$
|2 \alpha-P / Q|<c_{0} / Q^{2}
$$

that is,

$$
|\alpha-(2 k+1) / 2 Q|<c_{1} /(2 Q)^{2},
$$

where $c_{0}$ and $c_{1}$ are absolute constants. Now let

$$
s:=s_{q}=\log L+(2 k+1) \pi i / \alpha,
$$

where

$$
-L^{\alpha}-\alpha L+\alpha-1=0 \quad \text { and } \quad 0<L<1
$$

(clearly such an $L$ exists). Then

$$
s=\log L+2 \pi i Q+O(1 / Q)
$$

and

$$
h(s)=-L^{\alpha}-\alpha L \exp \{O(1 / Q\}+\alpha-1 \rightarrow 0 \text { as } Q \rightarrow \infty .
$$

Hence $h(s)$ assumes arbitrarily small values in the fixed half plane $\operatorname{Re}(z) \leq \log L<0$. Thus, by a theorem on exponential polynomials (see, e.g., [2, p. 403, Theorem 12.6]), $h(s)$ has infinitely many zeros in this half plane.

Proof of Theorem 4.1. With $s=\lambda u$ and $\alpha=1 / \lambda$ we have by Lemma 4.2 that

$$
\lambda e^{u}+1-\lambda-e^{\lambda u}=0
$$


has infinitely many roots with $\operatorname{Re}(u)<0$. Let $u_{0}$ be one of these. Then by Hurwitz's theorem there is an infinite sequence $u_{0}(n) \rightarrow u_{0}$ such that $u_{0}(n)$ is a root of

$$
\lambda\left(1+\frac{u}{n}\right)^{n}+1-\lambda=\left(1+\frac{\lambda u}{n}\right)^{n} .
$$

Fix $\delta>0$ such that $\pi / 2+\delta<\arg \left(u_{0}\right)<3 \pi / 2-\delta$. Then for $n$ sufficiently large, all $u_{0}(n)$ satisfy

$$
\frac{\pi}{2}+\delta<\arg \left(u_{0}(n)\right)<\frac{3 \pi}{2}-\delta
$$

If

$$
w_{n}:=1+\frac{u_{0}(n)}{n},
$$

then $\left|w_{n}\right|<1$ for $n$ sufficiently large, and $p_{n}(z)=0$ is satisfied for $z=w_{n}$. This completes the proof.

5. $\lambda$ an integer reciprocal. We formulate the necessity part of Theorem 1.1 as a separate theorem.

THEOREM 5.1. If $\lambda$ is the reciprocal of a positive integer greater than 2 , then apart from a double zero at $z=1$ every zero of $p_{n}(z)$, for $n \geq 475$, has modulus greater than 1 .

For $q=1 / \lambda \geq 4$ the lower bound of $n=475$ can be improved; see the remark at the end of this section.

We supplement Theorem 5.1 with the following result.

Proposition 5.2. For $\lambda=1 / 2$, the zeros of $p_{n}(z)(n \geq 2)$ all lie on the unit circle.

Proof. It is clear that for $\lambda=1 / 2$ we have

$$
p_{n}(z)=\frac{1}{2} z^{n}+\frac{1}{2}-\left(\frac{1}{2} z+\frac{1}{2}\right)^{n}=z^{n} p_{n}\left(\frac{1}{z}\right)
$$

i.e., $p_{n}(z)$ is a self-inverse polynomial. Hence by a theorem of A. Cohn (see, e.g., [9, p. 206]) the zeros of $p_{n}(z)$ lie on $U$ if and only if the zeros of the derivative all lie on or inside $U$. But this is easy to establish.

Theorem 5.1 is a consequence of the following somewhat stronger result.

THEOREM 5.3. For $\lambda$ the reciprocal of an integer $q \geq 3$, the image of $U$ under $p_{n}(z)$, for $n \geq 475$, intersects the nonpositive real axis $L$ only at $z=0$.

The principle of the argument and Theorem 5.3 now immediately imply that $p_{n}(z)$ cannot have any zeros inside $U$.

To prove Theorem 5.3, we rewrite $p_{n}\left(e^{i \theta}\right)$ as follows. Let

$$
\rho=\rho(\theta):=\left|\lambda e^{i \theta}+1-\lambda\right|^{n}
$$

and

$$
\sigma=\sigma(\theta):=n \arg \left(\lambda e^{i \theta}+1-\lambda\right)
$$


so that we can rewrite $p_{n}\left(e^{i \theta}\right)$ as

$$
p_{n}\left(e^{i \theta}\right)=\lambda e^{i n \theta}+1-\lambda-\rho(\theta) e^{i \alpha(\theta)} .
$$

First we note that whenever $\rho(\theta)<1-2 \lambda$, the curve $p_{n}\left(e^{i \theta}\right)$ is confined to the interior of a circle of radius $1-\lambda$ centered at $1-\lambda$; hence it does not intersect the negative real axis in this case. We may therefore restrict our attention to the case

$$
\rho(\theta) \geq 1-2 \lambda \text {. }
$$

The main idea of proof now is to study the related curve

$$
f_{\rho(\theta)}(n \lambda \theta)=\lambda e^{i n \theta}+1-\lambda-\rho(\theta) e^{i n \lambda \theta}, \quad 0 \leq \theta \leq 2 \pi,
$$

and to show that (5.5) approximates (5.3) quite well, under the condition (5.4). Introduce the function of two variables

$$
f_{\rho}(t):=\lambda e^{i t / \lambda}+1-\lambda-\rho e^{i t}
$$

Let $E=E(\rho)$ be, for a fixed $\rho$ with $0 \leq \rho \leq 1$, the curve $z=f_{\rho}(t)$ where $t \geq 0$. We shall show the following results.

Proposition 5.4. The point on the negative real axis $L$ closest to $E(\rho)$ is the origin.

Proposition 5.5. For $0 \leq t \leq 2 \pi$ we have

$$
\left|f_{\rho}(t)\right| \geq \frac{C}{\sqrt{\lambda}}(1-\rho)^{3 / 2},
$$

where

$$
C=\left\{\begin{array}{lll}
\frac{1}{6}\left(1-\frac{1}{2} \sqrt{2}\right) & \text { if } & q=3, \\
(1-2 \lambda) / 4 \sqrt{2} & \text { if } & 4 \leq q \leq 15, \\
7 / 32 \sqrt{2} & \text { if } & q \geq 16 .
\end{array}\right.
$$

Proposition 5.6. For $\rho(\theta)$ as in (5.4) and for $n \geq 16$ we have

$$
\left|f_{\rho(\theta)}(n \lambda \theta)-p_{n}\left(e^{i \theta}\right)\right| \leq \frac{11 / 19}{\sqrt{\lambda}(1-\lambda)^{3 / 2} \sqrt{n}}(1-\rho(\theta))^{3 / 2} .
$$

Now every point $p_{n}\left(e^{i \theta}\right)$ is close to an $f_{\rho(\theta)}(n \lambda \theta)$, and this in turn is on a curve $f_{\rho(\theta)}(t)$, every point of which is at least a distance of

$$
\left|f_{\rho}(t)-0\right|=\left|f_{\rho}(t)\right| \geq \frac{C}{\sqrt{\lambda}}(1-\rho)^{3 / 2}
$$

from $L$, by Propositions 5.4 and 5.5. Hence we know that $p_{n}\left(e^{i \theta}\right)$ avoids $L$ provided that the right-hand side of (5.7) is less than the right-hand side of (5.6). But this is the case when

$$
n \geq\left(\frac{11 / 19}{C(1-\lambda)^{3 / 2}}\right)^{2}=\frac{121 / 361}{C^{2}(1-\lambda)^{3}} .
$$

In particular, for $q=3$ (i.e., $\lambda=1 / 3),(5.8)$ holds for

$$
n \geq \frac{121 \times 9 \times 27}{361(3-2 \sqrt{2})} \cong 474.718 \text {. }
$$


For $4 \leq q \leq 15$ we need

$$
n \geq \frac{32 \times 121 / 361}{(1-2 \lambda)^{2}(1-\lambda)^{3}}(\cong 101.696 \text { for } q=4)
$$

and the case $q>16$ requires

$$
n \geq \frac{121 \times 2048}{361 \times 49(1-\lambda)^{3}}(\cong 17.002 \text { for } q=16) .
$$

This completes the proof of Theorem 5.1, and actually a little more.

Remark. We can use Theorem 3.1 and some additional arguments to further improve the bounds on $n$ in Theorem 5.1.

First we note, using (5.9), that Theorem 5.1 holds for all $n \geq 16$ provided that $q \geq 24$. Now by Theorem $3.1, p_{n}(z)$ has no zero inside $U$ for $n \leq 15$ provided that $q \geq 143$, or for $n \leq 14$ if $q \geq 133$, etc., down to $n \leq 10$ if $q \geq 92$. This means that for $q \geq 143$, Theorem 5.1 holds for all $n$, while for $133 \leq q \leq 142$ the case $n=15$ remains open, for $123 \leq q \leq 132$ the cases $n=14$ and $n=15$ remain open, etc. These gaps can be closed by deriving explicit expressions for the coefficients of the polynomials $p_{n}(z) /(z-1)^{2}$ and observing that they form decreasing sequences in the cases $n=15$ and $95 \leq q \leq 142$, $n=14$ and $83 \leq q \leq 132$, etc. Lemma 2.3 now implies that the zeros of $p_{n}(z)$ different from 1 lie outside $U$ in the above cases. (Note that this may be considered as the quantitative version of the remark at the end of Section 3.) We have now

Proposition 5.7. Theorem 5.1 is true for all $n \geq 2$ when $q=1 / \lambda \geq 95$.

This bound of $q=95$ could be further improved by using generalizations of the Eneström-Kakeya theorem (see [4]). We suspect that numerical methods could close the remaining gaps.

6. Proof of Proposition 5.4. For $\lambda=1 / q$, where $q \geq 3$ is an integer, we shall study the curve $E=E(\rho)$ given by the function

$$
f_{\rho}(t)=\lambda e^{i t / \lambda}+1-\lambda-\rho e^{i t}, \quad 0 \leq \rho \leq 1 .
$$

Since $1 / \lambda$ is an integer, we need to consider $f_{\rho}(t)$ only for $0 \leq t \leq 2 \pi$. For $\rho=1, f_{\rho}(t)$ has a zero at $t=0$.

LEMma 6.1. If $\rho<1$, the curve $E$ does not intersect $L$.

Proof. Since $f_{\rho}(0)=f_{\rho}(2 \pi)=1-\rho>0$ and $f_{\rho}(\pi) \geq 1-2 \lambda+\rho>0$, we may assume that $\sin t \neq 0$. Decompose $f_{\rho}(t)$ into its real and imaginary parts. If the imaginary part $y=0$, then $\rho=\sin q t / q \sin t$ and consequently

$$
q \operatorname{Re}\left(f_{\rho}(t)\right)=\cos q t+q-1-\sin q t \frac{\cos t}{\sin t}=(q-1)-\frac{\sin (q-1) t}{\sin t}>0,
$$

by a well-known inequality that can be verified by induction on $q$. This proves the lemma.

To show that the origin is the point on $L$ closest to the curve $E$, we shall study some geometric properties of $E$. In fact, $E$ is an epitrochoid (see, e.g., [18, p. 233]). If we 
rewrite

$$
f_{\rho}(t)=1-\lambda-\rho e^{i t}\left(1-\frac{1}{q \rho} e^{i(q-1) t}\right),
$$

it becomes clear that $E$ can be thought of as the trajectory of a particle on a circular orbit of radius $1 / \rho q$ and frequency $q-1$, superimposed on another circular orbit of radius $\rho$ and frequency 1 . (6.2) also shows that $E$ has $q-1$ fold symmetry about its center.

Lemma 6.2. Suppose that $\rho<1$ and that (5.4) holds. Then

(a) $E$ is confined to the annulus with center $1-\lambda$, inner radius $\rho-\lambda$ and outer radius $\rho+\lambda$;

(b) $E$ is symmetric about all straight lines through its center making angles $k \pi /(q-1)$, $k=0,1, \ldots, q-2$ with the real axis;

(c) the tangent vector to $E$ has an argument that increases monotonically with $t$;

(d) the net change in argument of the tangent vector is $2 \pi q$ as $t$ goes from 0 to $2 \pi$. by (5.4).

Proof. (a) This is clear from (6.1) if we note that $\lambda=1 / q \leq 1 / 3$ and $\rho \geq 1-2 \lambda \geq 1 / 3$,

(b) Let

$$
g(t):=f\left(\frac{k \pi}{q-1}+t\right) e^{-i k \pi /(q-1)} .
$$

Then it is easy to verify that $g(-t)=\overline{g(t)}$. This proves the symmetry about the line in question.

(c) We split $f_{\rho}(t)$ into its real, resp. imaginary components

$$
\begin{aligned}
R(t) & :=\lambda \cos q t-\rho \cos t+1-\lambda, \\
I(t) & :=\lambda \sin q t-\rho \sin t
\end{aligned}
$$

The tangent vector

$$
f_{\rho}^{\prime}(t)=i e^{i q t}-\rho i e^{i t}
$$

has components

$$
\begin{aligned}
R^{\prime}(t) & =-\sin q t+\rho \sin t, \\
I^{\prime}(t) & =\cos q t-\rho \cos t .
\end{aligned}
$$

If $\theta(t)$ is the argument of the tangent vector, then $\tan \theta(t)$ has derivative

$$
\frac{d}{d t} \tan \theta(t)=\frac{q+\rho^{2}-\rho(1+q) \cos (q-1) t}{(\sin t q-\rho \sin t)^{2}} \geq \frac{(1-\rho)(q-\rho)}{(\sin t q-\rho \sin t)^{2}}>0
$$

the last inequality follows from $0<\rho<1$ and $q \geq 3$. This proves (c). (d) is clear from (6.5) and the fact that $\rho<1$.

Proof of Proposition 5.4. Because of the $q-1$ fold symmetry about the center $z=1-\lambda$ of $E$, we need only consider $0 \leq t \leq 2 \pi /(q-1)$. From (6.3), (6.4), (6.6) and (6.7) we see that at $t_{0}=0, E$ passes through $z=1-\rho$ and has a vertical tangent there. As $t$ increases, $E$ then moves to the left in the first quadrant until it reaches a point $t_{1}$ with 
horizontal tangent, where $0<t_{1}<\pi / 2 q$ (by (6.7)). Then for some $t_{2}$ with $\pi / 2 q<t_{2}<\pi / q$ (by (6.4)), $E$ cuts the positive $x$-axis (positive because of Lemma 6.1) between $z=0$ and $z=1-\rho$. This has to be a double point since $E$ is symmetric about the real axis. Furthermore, because of the $q-1$ fold symmetry about the center of $E$, another double point occurs on the ray $R$ emanating from the center of $E$ with argument $\pi+2 \pi /(q-1)$. This will occur for some $t_{3}$ with $2 \pi /(q-1)-\pi / q<t_{3}<2 \pi /(q-1)$. Next, by Lemma 6.2(b) with $k=1$ the tangent of $E$ is parallel to $R$ for some $t_{4}, 2 \pi /(q-1)-\pi / 2 q<t_{4}<$ $2 \pi /(q-1)$. Finally, $E$ cuts $R$ at a right angle for $t_{5}=2 \pi /(q-1)$. Now the same process starts again, with the real axis replaced by $R$.

Hence we may consider $E$ as consisting of a union $E^{\prime}$ of curve segments between consecutive double points, and a union $E^{\prime \prime}$ of loops that lie in the interior of the piecewise differentiable curve $E^{\prime}$.

Now we consider a "slice", namely the interior of the closed curve consisting of a segment of $E^{\prime}$ between two consecutive double points, and the straight line segments joining the center of $E$ with these double points. Then $E^{\prime}$ together with its interior is the union of all these $q-1$ slices. By Lemma 6.2(d), the change in argument of the tangent vector between $t=t_{0}$ and $t=t_{5}$ is $2 \pi q /(q-1)$ and therefore between $t=t_{1}$ and $t=t_{4}$ it is $2 \pi q /(q-1)-2(\pi / 2)=\pi+2 \pi /(q-1)$; as a consequence, there can be no other double point between $t_{2}$ and $t_{3}$. This, together with Lemma $6.2(\mathrm{c})$, shows that each of the $q-1$ slices is convex.

Next we note that because of symmetry, each point $z_{0}$ on the nonpositive real axis $L$ has the same distance to the two slices lying just above and just below the real axis and closest to the origin, and that the distance to any other slice is greater. Hence the distance of $z_{0}$ to all of $E$ is the same as the distance to one of the two slices mentioned above.

Finally we use the fact that the distance between a closed convex region and a point moving along a straight line is a unimodal function, i.e., it is nonincreasing up to a certain point, and then it is nondecreasing. The proposition is proved if we apply this to the slice and the real axis.

7. Proof of Proposition 5.5. First we note that because of $f_{\rho}(2 \pi-t)=\overline{f_{\rho}(t)}$ we may restrict our attention to $0 \leq t \leq \pi$. Furthermore, we claim that $\left|f_{\rho}(t)\right|$ cannot be minimal for $\pi / 2 \leq t \leq \pi$. Indeed, consider

$$
\left|f_{\rho}(t)\right|^{2}=\lambda^{2}+\rho^{2}+(1-\lambda)^{2}-2 \rho \lambda \cos (q-1) t+2(1-\lambda)(\lambda \cos q t-\rho \cos t)
$$

which follows from (6.3) and (6.4). With $\pi / 2 \leq t \leq \pi$ we get

$$
\left|f_{\rho}(t)\right|^{2} \geq 1-4 \lambda+4 \lambda^{2}+\rho^{2}-2 \rho \lambda
$$

The right-hand side of this is greater than or equal to $\left|f_{\rho}(0)\right|^{2}=(1-\rho)^{2}$ if and only if $\rho \geq 2 \lambda$. But this is true whenever $\lambda \leq 1 / 4$ since we may assume $1-2 \lambda \leq \rho$ by (5.4). For the remaining case $\lambda=1 / 3$ (i.e., $q=3$ ) we note that for $\pi / 2 \leq t \leq \pi$,

$$
\lambda \cos (q-1) t+(1-\lambda) \cos t=\frac{2}{3}\left(\cos ^{2} t+\cos t\right)-\lambda \leq-\lambda,
$$

so that in this case, by (7.1),

$$
\left|f_{\rho}(t)\right|^{2} \geq 1-4 \lambda+4 \lambda^{2}+\rho^{2}+2 \rho \lambda
$$


The right-hand side of this is greater than or equal to $\left|f_{\rho}(0)\right|^{2}=(1-\rho)^{2}$ if and only if $\rho \geq 2 \lambda(1-\lambda) /(1+\lambda)=1 / 3$, which holds because of $\rho \geq 1-2 \lambda$.

Hence we may restrict our attention to $0 \leq t \leq \pi / 2$. Now it is easy to see that Proposition 5.5 is a consequence of the following result.

Proposition 7.1. Let $C:=5 / 4 \sqrt{q}$, and

$$
\begin{aligned}
& I_{1}: 0 \leq t \leq C \sqrt{1-\rho} \\
& I_{2}: C \sqrt{1-\rho} \leq t \leq \pi / 2(q-1), \\
& I_{3}: \pi / 2(q-1) \leq t \leq \pi / 2
\end{aligned}
$$

Then

$$
\left|f_{\rho}(t)\right| \geq D \sqrt{q}(1-\rho)^{3 / 2}
$$

where

$$
D:= \begin{cases}7 / 32 \sqrt{2} & \text { for } t \in I_{1}, \\ 557(1-\lambda)(1-2 \lambda) / 2048 & \text { for } t \in I_{2}, \\ (1-2 \lambda) / 4 \sqrt{2} & \text { for } t \in I_{3} \text { and } q \geq 4, \\ (\sqrt{2}-1) / 6 \sqrt{2} & \text { for } t \in I_{3} \text { and } q=3 .\end{cases}
$$

Proof. Differentiating (7.1) with respect to $t$, we find that $\left|f_{\rho}(t)\right|^{2}$ is minimal (at least for $t>0$ ) when

$$
\rho=\frac{\sin q t}{\sin (q-1) t+\sin t}
$$

which implies

$$
\lambda \sin q t-\rho \sin t=\lambda \rho[\sin (q-1) t-(q-1) \sin t] .
$$

Using the estimate

$$
\sin \frac{\pi}{2(q-1)} \geq \frac{\pi}{2(q-1)}-\frac{1}{6}\left(\frac{\pi}{2(q-1)}\right)^{3}
$$

we get for $t \in I_{3}$ and $q \geq 3$ that

$$
|\sin (q-1) t-(q-1) \sin t| \geq\left|(q-1) \sin \frac{\pi}{2(q-1)}-1\right| \geq C^{\prime},
$$

where $C^{\prime}=1 / 2$ for $q \geq 4$ and $C^{\prime}=\sqrt{2}-1$ for $q=3$. Hence with (6.4) and (7.3) we have

$$
\left|f_{\rho}(t)\right| \geq I(t)>C^{\prime} \lambda \rho \text {. }
$$

Using $1-2 \lambda \leq \rho$ twice, we get

$$
C^{\prime} \lambda \rho=\frac{C^{\prime}}{2 \sqrt{2}}(2 \lambda)^{3 / 2} \sqrt{q} \rho \geq \frac{C^{\prime}}{2 \sqrt{2}}(1-\rho)^{3 / 2} \sqrt{q}(1-2 \lambda),
$$

so that with (7.4) we have

$$
\left|f_{\rho}(t)\right|>\frac{C^{\prime}}{2 \sqrt{2}}(1-2 \lambda) \sqrt{q}(1-\rho)^{3 / 2} \quad \text { for } \quad t \in I_{3}
$$


Next we consider the interval $\left(I_{2}\right)$. With (6.3) and (6.4) we find

$$
R(t) \sin t-I(t) \cos t=\frac{q-1}{q}\left[\sin t-\frac{1}{q-1} \sin (q-1) t\right] .
$$

We denote the expression in brackets by $h(t)$ and observe that $h(t)$ is increasing for $0 \leq t \leq \pi / 2(q-1)$. Hence for $t$ in the interval $\left(I_{2}\right)$ we have, using a Taylor expansion,

$$
\begin{aligned}
h(t) \geq & \sin (C \sqrt{1-\rho})-\frac{1}{q-1} \sin [(q-1) C \sqrt{1-\rho}] \\
\geq & C \sqrt{1-\rho}-\frac{1}{6}(C \sqrt{1-\rho})^{3}-\frac{1}{q-1}\left[(q-1) C \sqrt{1-\rho}-\frac{1}{6}((q-1) C \sqrt{1-\rho})^{3}\right. \\
& \left.+\frac{1}{120}((q-1) C \sqrt{1-\rho})^{5}\right] \\
= & \frac{1}{6} q(q-2)(C \sqrt{1-\rho})^{3}\left\{1-\frac{1}{20} \frac{(q-1)^{2}}{q(q-2)}[(q-1) C \sqrt{1-\rho}]^{2}\right\} .
\end{aligned}
$$

Using the facts that $(q-1)^{2} / q(q-2) \leq 4 / 3$ for $q \geq 3$, and that $(q-1) C \sqrt{1-\rho} \leq \pi / 2$, we get

$$
h(t) \geq \frac{557}{4000} q(q-2) C^{3}(1-\rho)^{3 / 2}
$$

and by (7.6) we have

$$
\begin{aligned}
\left|f_{\rho}(t)\right| & =\left(|R(t)|^{2}+|I(t)|^{2}\right)^{1 / 2} \\
& \geq|R(t) \sin t-I(t) \cos t| \\
& =\frac{q-1}{q}|h(t)| \geq \frac{557}{4000}(q-1)(q-2) C^{3}(1-\rho)^{3 / 2} \text { for } \quad t \in I_{2} .
\end{aligned}
$$

Finally, we consider the interval $I_{1}$. Using (6.3) and a Taylor expansion, we find

$$
\begin{aligned}
R(t) & \geq \lambda\left(1-\frac{1}{2}(q t)^{2}\right)-\rho\left(1-\frac{1}{2} t^{2}+\frac{1}{24} t^{4}\right)+1-\lambda \\
& =1-\rho-\frac{1}{2} q t^{2}+\frac{1}{2} \rho t^{2}\left(1-\frac{1}{12} t^{2}\right) \\
& \geq 1-\rho-\frac{1}{2} q t^{2} .
\end{aligned}
$$

Now we use the restrictions $t^{2} \leq C^{2}(1-\rho)$ and $1-2 \lambda \leq \rho$ to get

$$
R(t) \geq\left(1-\frac{1}{2} q C^{2}\right)(1-\rho)
$$

and

$$
\left|f_{\rho}(t)\right| \geq R(t) \geq\left(1-\frac{1}{2} q C^{2}\right) \frac{1}{\sqrt{2}} \sqrt{q}(1-\rho)^{3 / 2} \text { for } t \in I_{1} .
$$

Now Proposition 7.1 follows from (7.5), (7.7) and (7.8).

8. Proof of Proposition 5.6. We begin with two lemmas. The first one shows, among other things, that only for small arguments $\theta$ in (5.3) does the condition (5.4) hold. Lemma 8.2 then states that for these $\theta$, the exponent $\operatorname{in} \lambda \theta$ in (5.5) is a good approximation of $i \sigma(\theta)$ in (5.3), and this leads to Proposition 5.6. 
LeMMA 8.1. Under condition (5.4) we have for $0 \leq \theta \leq \pi$ and $n \geq 16$,

$$
\begin{gathered}
\theta^{2} \leq 4 \frac{d}{n}\left(1+\frac{3}{8} \frac{d}{n}\right), \text { where } d:=\left(1+\lambda+3 \lambda^{2}\right) /(1-\lambda) ; \\
\rho(\theta) \leq \exp \left\{-\frac{17}{36} \lambda(1-\lambda) n \theta^{2}\right\} .
\end{gathered}
$$

Proof. From (5.1) we get

$$
\begin{aligned}
\rho(\theta)^{2 / n} & =1+2 \lambda(1-\lambda)(\cos \theta-1) \\
& \leq 1+2 \lambda(1-\lambda)\left(-\frac{1}{2} \theta^{2}+\frac{1}{24} \theta^{4}\right) \\
& =1-\lambda(1-\lambda) \theta^{2}\left(1-\frac{1}{12} \theta^{2}\right),
\end{aligned}
$$

and therefore

$$
\rho(\theta)^{2 / n} \leq 1-C \theta^{2}
$$

with

$$
C=C_{\theta}:=\lambda(1-\lambda)\left(1-\theta^{2} / 12\right) .
$$

Using (5.4) and (8.3), we find

$$
\frac{1}{n} \log (1-2 \lambda) \leq \log \rho^{1 / n} \leq \frac{1}{2} \log \left(1-C \theta^{2}\right) \leq-\frac{1}{2} C \theta^{2} .
$$

This implies

$$
\begin{aligned}
\frac{1}{2} C \theta^{2} & \leq \frac{1}{n} \log \frac{1}{1-2 \lambda}=\frac{1}{n}\left[2 \lambda+\frac{1}{2}(2 \lambda)^{2}+\ldots\right] \\
& \leq \frac{1}{n}\left[2 \lambda+2 \lambda^{2}+\frac{1}{3}(2 \lambda)^{3}\left(1+2 \lambda+(2 \lambda)^{2}+\ldots\right)\right] \\
& =\frac{2 \lambda}{n}\left[1+\lambda+\frac{4}{3} \lambda^{2}(1-2 \lambda)^{-1}\right] \\
& \leq \frac{2 \lambda}{n}\left(1+\lambda+3 \lambda^{2}\right),
\end{aligned}
$$

and with (8.4) we get

$$
\theta^{2}\left(1-\theta^{2} / 12\right) \leq 4 d / n
$$

Since $n \geq 16$ this implies

$$
\theta^{2} \leq 6-6 \sqrt{1-4 d / 3 n}
$$

It is easy to check that

$$
\sqrt{1-4 d / 3 n} \geq 1-\frac{2}{3} \frac{d}{n}-\frac{1}{4} \frac{d^{2}}{n^{2}}
$$

where we have used the fact that $d \leq 5 / 2$ for $\lambda \leq 1 / 3$, and consequently $d / n \leq 5 / 32$ for $n \geq 16$. (8.1) now follows from (8.6). 
To verify (8.2), we note that with (8.5) and (8.4) we have

$$
\rho \leq \exp \left\{-\frac{1}{2} \lambda(1-\lambda)\left(1-\frac{\theta^{2}}{12}\right) n \theta^{2}\right\} .
$$

Now by (8.1), since $d / n \leq 5 / 32$,

$$
\frac{\theta^{2}}{12} \leq \frac{1}{3} \frac{d}{n}\left(1+\frac{5}{3} \frac{d}{n}\right) \leq \frac{1}{18}
$$

and this proves (8.2).

LEMMA 8.2. For $\theta$ as in (8.1) and for $n \geq 16$ we have

$$
|\sigma(\theta)-n \lambda \theta| \leq \frac{\lambda}{6} n \theta^{3} .
$$

Proof. With (5.2) we get

$$
\tan \frac{\sigma}{n}=\frac{\lambda \sin \theta}{\lambda \cos \theta+1-\lambda} .
$$

Using Taylor expansions for sine and cosine, we get

$$
\begin{aligned}
\tan \frac{\sigma}{n} & \leq \lambda\left(\theta-\frac{1}{6} \theta^{3}+\frac{1}{120} \theta^{5}\right)\left(1-\frac{\lambda}{2} \theta^{2}\right)^{-1} \\
& \leq \lambda \theta\left(1-\frac{1}{6} \theta^{2}+\frac{1}{120} \theta^{4}\right)\left(1-\frac{1}{6} \theta^{2}\right)^{-1} \\
& =\lambda \theta\left[1+\frac{\theta^{4}}{120} \frac{1}{1-\theta^{2} / 6}\right] .
\end{aligned}
$$

With (8.7) we easily see that

$$
\tan \frac{\sigma}{n} \leq \lambda \theta+\frac{1}{6} \lambda \theta^{3}
$$

and with the Taylor expansion for the arctangent we get

$$
\frac{\sigma}{n} \leq \lambda \theta+\frac{1}{6} \lambda \theta^{3}
$$

In the other direction, we find with (8.8) that

$$
\begin{aligned}
\tan \frac{\sigma}{n} & \geq \lambda\left(\theta-\frac{1}{6} \theta^{3}\right)\left(1-\frac{\lambda}{2} \theta^{2}+\frac{\lambda}{24} \theta^{4}\right)^{-1} \\
& =\lambda \theta\left(1-\frac{1}{6} \theta^{2}\right)\left[1-\frac{1}{2} \lambda \theta^{2}\left(1-\theta^{2} / 12\right)\right]^{-1} \\
& \geq \lambda \theta\left(1-\frac{1}{6} \theta^{2}\right)\left(1-\frac{15}{32} \lambda \theta^{2}\right)^{-1} \\
& \geq \lambda \theta\left(1-\frac{1}{6} \theta^{2}\right)\left(1+\frac{15}{32} \lambda \theta^{2}\right) \\
& =\lambda \theta-\frac{\lambda}{6} \theta^{3}\left[1-\frac{45}{16} \lambda\left(1-\frac{\theta^{2}}{6}\right)\right]
\end{aligned}
$$


Using (8.7) again, we find

$$
\tan \frac{\sigma}{n} \geq \lambda \theta-\frac{\lambda}{6} \theta^{3}(1-2 \lambda)
$$

and with a Taylor expansion for the arctangent, we get

$$
\begin{aligned}
\frac{\sigma}{n} & \geq \lambda \theta-\frac{\lambda}{6} \theta^{3}(1-2 \lambda)-\frac{1}{3}\left[\lambda \theta-\frac{\lambda}{6} \theta^{3}(1-2 \lambda)\right]^{3} \\
& \geq \lambda \theta-\frac{\lambda}{6} \theta^{3}\left(1-2 \lambda+2 \lambda^{2}\right) \\
& \geq \lambda \theta-\frac{\lambda}{6} \theta^{3},
\end{aligned}
$$

and this, together with (8.9), proves the lemma.

Proof of Proposition (5.6). With (5.3) and (5.5) we get

$$
\left|f_{\rho(\theta)}(n \lambda \theta)-p_{n}\left(e^{i \theta}\right)\right| \leq \rho(\theta)\left|\int_{n \lambda \theta}^{\sigma(\theta)} e^{i t} d t\right| \leq \rho(\theta)|\sigma(\theta)-n \lambda \theta|,
$$

and by Lemma 8.2 ,

$$
\left|f_{\rho(\theta)}(n \lambda \theta)-p_{n}\left(e^{i \theta}\right)\right| \leq \frac{1}{6} \rho \lambda n \theta^{3} .
$$

From the first part of Lemma 8.1 and the bounds $\lambda(1-\lambda) \leq 2 / 9, d \leq 5 / 2$, and (for $n \geq 16$ ) $d / n \leq 5 / 32$ we find that

$$
n \theta^{2} \leq 4 d\left(1+\frac{3 d}{8 n}\right) \leq \frac{108}{10} .
$$

Now the Taylor expansion of the exponential and the second part of Lemma 8.1 yield

$$
\begin{aligned}
\rho^{-2 / 3}-\rho^{1 / 3} & \geq \exp \left\{\frac{17}{54} \lambda(1-\lambda) n \theta^{2}\right\}-\exp \left\{-\frac{17}{108} \lambda(1-\lambda) n \theta^{2}\right\} \\
& \geq 1+\frac{17}{54} \lambda(1-\lambda) n \theta^{2}-\left\{1-\frac{17}{108} \lambda(1-\lambda) n \theta^{2}+\frac{1}{2}\left[\frac{17}{108} \lambda(1-\lambda) n \theta^{2}\right]^{2}\right\} \\
& =\frac{51}{108} \lambda(1-\lambda) n \theta^{2}\left\{1-\frac{17}{648} \lambda(1-\lambda) n \theta^{2}\right\} .
\end{aligned}
$$

Another application of the above estimates gives us

$$
\rho^{-2 / 3}-\rho^{1 / 3} \geq \frac{11}{25} \lambda(1-\lambda) n \theta^{2} .
$$

This implies

$$
\rho \lambda n \theta^{3} \leq\left(\frac{25}{11}\right)^{3 / 2} \frac{1}{\sqrt{\lambda}(1-\lambda)^{3 / 2} \sqrt{n}}(1-\rho)^{3 / 2}
$$

this, with $(8.10)$, proves the proposition if we note that $(25 / 11)^{3 / 2} / 6 \leq 11 / 19$.

\section{REFERENCES}

1. E. F. Beckenbach and R. E. Bellman, Inequalities (Springer, Berlin, 1961).

2. R. E. Bellman and K. L. Cooke, Differential-difference equations (Academic Press, New York 1963). 
3. G. Boese, Einschlüsse und Trennung der Nullstellen von Exponentialtrinomen, Z. Angew. Math. Mech. 62 (1982), 547-560.

4. K. Dilcher, A generalization of the Eneström-Kakeya theorem, J. Math. Anal. Appl. 116 (1986), 473-488.

5. K. Dilcher and K. B. Stolarsky, Zeros of the Wronskian of a polynomial, J. Math. Anal. Appl. (to appear).

6. R. J. Evans and K. B. Stolarsky, A family of polynomials with concyclic zeros II, Proc. Amer. Math. Soc. 92 (1984) 393-396.

7. G. H. Hardy, J. E. Littlewood, and G. Pólya, Inequalities (Cambridge Univ. Press, 1964).

8. E. Laguerre, Oeuvres, Vol. 1, 2nd ed. (Chelsea Publishing, New York, 1972).

9. M. Marden, Geometry of polynomials, Mathematical Surveys (3), (Amer. Math. Soc., Providence 1966).

10. J. L. Nicolas and A. Schinzel, Localisation des zeros de polynômes intervenant en théorie du signal. In: M. Langevin and M. Waldschmidt (Eds.), Cinquante Ans de Polynômes. Lecture Notes in Mathematics 1415, Springer-Verlag 1990.

11. J. D. Nulton and K. B. Stolarsky, Zeros of certain trinomials, C. R. Math. Rep. Acad. Sci. Canada 6 (1984), 243-248.

12. G. Pólya and G. Szegö, Problems and Theorems in Analysis, I, 4th ed., (Springer Verlag, Berlin 1970). 332-349.

13. Q. I. Rahman and J. Szynal, On some classes of polynomials, Canad. J. Math. 30 (1978),

14. Q. I. Rahman and J. Waniurski, Coefficient regions for univalent trinomials, Canad. J. Math. 32 (1980), 1-20.

15. K. B. Stolarsky, Zeros of exponential polynomials and "reductionism", Topics in Classical Number Theory, Coll. Math. Soc. János Bolyai 34, Elsevier North Holland, Amsterdam 1984.

16. K. B. Stolarsky, A family of polynomials with concyclic zeros, Proc. Amer. Math. Soc. 88 (1983), 622-624.

17. K. B. Stolarsky, A family of polynomials with concyclic zeros III, Quart. J. Math. Oxford (2) 36 (1985), 255-259.

18. R. C. Yates, A Handbook on Curves and their Properties (J. W. Edwards, Ann Arbor 1959).

Dept. of Mathematics, Statistics and Computing Science

Dalhousie UNIVERsity

2045 Montclair Street

Halifax

SAN Diego

Nova ScOTIa

California 92104

Canada B3H $3 J 5$

Dept. of Mathematics

1409 WEST GREEN

UNIVERSITY OF ILLINOIS

URBANA, ILLINOIS 61801

U.S.A. 